\title{
Resistant dextrin reduces obesity and attenuates adipose tissue inflammation in high-fat diet-fed mice
}

\author{
Qiuyue $\mathrm{Hu}^{\#}$, Yao Lu\#, Fan $\mathrm{Hu}^{\#}$, Sunyue He, Xiaoyuan $\mathrm{Xu}$, Yixin Niu, Hongmei Zhang, Xiaoyong Li ${ }^{\bowtie}$ and \\ Qing $\mathrm{Su}^{\square}$ \\ Department of Endocrinology, Xinhua Hospital, Shanghai JiaoTong University School of Medicine, 1665 Kongjiang Road, Shanghai 200092, China. \\ \#These authors contributed equally to this work. \\ $\triangle$ Corresponding authors: Xiaoyong Li, E-mail: lixiaoyong0055@126.com, Tel.: 8613585603326; Qing Su, E-mail: suqing@xinhuamed.com.cn, Tel.: 8613918913783.
}

(C) The author(s). This is an open access article distributed under the terms of the Creative Commons Attribution License (https://creativecommons.org/licenses/by/4.0/). See http://ivyspring.com/terms for full terms and conditions.

Received: 2020.03.06; Accepted: 2020.08.31; Published: 2020.09.20

\begin{abstract}
Resistant dextrin (RD), a short chain glucose polymer, has been shown to improve type 2 diabetes mellitus (T2DM) in clinical studies. However, the improvement of adipose tissue inflammation and specific mechanisms of RD supplementation in obesity have not been fully investigated. Therefore, we examined whether RD attenuates obesity and adipose tissue inflammation in high-fat diet (HFD)-fed mice. Male C57BL/6 mice were fed a chow diet, a HFD or a HFD with RD supplementation for 12 weeks. Body weight (BW), fasting blood glucose (FBG), epididymal fat accumulation, serum total triglyceride (TG), free fatty acid (FFA) and inflammatory cytokine levels (TNF- $\alpha$, IL-1 $\beta$, IL-6, IL-10) were measured. Inflammation markers and macrophage infiltration in epididymal adipose tissue were observed. After 12 weeks of intervention, the body weight gain of mice in RD supplementation group was less than that in HFD group. FBG, epididymal fat accumulation, serum TG and FFA levels were reduced in RD supplementation group compared with HFD group. Moreover, serum and mRNA levels of IL- 6 were significantly reduced in the RD supplementation group. In addition, RD supplementation reduced macrophage infiltration, regulated polarization of macrophage and inhibited NF-KB signaling in epididymal adipose tissue. In conclusion, RD reduces obesity and attenuates adipose tissue inflammation in HFD-fed mice, and the inhibition of NF-KB signaling may be a presumed mechanism for its effects.
\end{abstract}

Key words: Resistant dextrin, Obesity, Adipose tissue inflammation, NF-kB signaling pathway

\section{Introduction}

Obesity, a worldwide epidemic, has contributed to a series of health problems for both individuals and society [1]. Obesity involves the excess accumulation of body fat and may contribute to multiple metabolic diseases, including type 2 diabetes, cardiovascular disease, fatty liver disease, cancers and stroke [1-5]. In addition, obesity increases the risk of joint pain, arthritis, sleep apnea and mental disorders [2, 6]. Therefore, effective treatment measures are urgently needed for this global health problem. Diet, exercise, anti-obesity drugs and bariatric surgery are the main approaches to prevent and treat obesity [7]. However, lifestyle modifications and drugs are often not largely effective in preventing obesity and maintaining weight loss over time [7, 8]. Although bariatric surgery is largely beneficial to weight loss and reduces adverse health consequences [5, 8-10], longterm health outcomes of bariatric surgery are regrettably unclear and it is not accessible to people in low- and middle-income countries [11]. Thus, a novel intervention strategy is crucial to solve or alleviate this major global problem.

Dietary fiber, defined as carbohydrate polymers that are neither digested nor absorbed in the human small intestine, is obtained from food raw materials and has physiological benefits [12]. Previous studies have demonstrated that dietary fiber is negatively associated with the risk of dyslipidemia, hypertension, obesity, diabetes, peripheral vascular disease, coronary heart disease and stroke [13]. Resistant dextrin (RD) is a soluble dietary fiber and prebiotic, which has been widely used in functional 
food and beverage products due to its low viscosity and great water solubility $[12,14,15]$. RD is rich in $\mathrm{a}-1,4$ and $a-1,6$ linkages and derives from wheat or maize [14, 16]. In addition, RD partially resists enzymatic hydrolysis in the human gastrointestinal tract $[14,17]$ : approximately $15 \%$ of RD is digested in the small intestine, $75 \%$ is fermented in the colon, and approximately $10 \%$ is excreted in the feces [18]. Short-term and long-term gastrointestinal tolerance studies of RD have shown that the fiber supplementation is well tolerated up to a dose of $45 \mathrm{~g}$ daily in healthy men $[19,20]$.

Moreover, RD supplementation was shown to regulate metabolic parameters and androgen levels in women with polycystic ovary syndrome (PCOS) [18]. RD supplementation has also been shown to improve magnesium and calcium absorption and retention [21]. Although a previous study has shown that body weight is reduced in overweight men who are given $17 \mathrm{~g}$ RD twice daily [22], the improvement of adipose tissue inflammation and specific mechanisms of RD supplementation in HFD-fed induced obese mice are not still investigated.

In the present study, our results show that RD reduces obesity and attenuates adipose tissue inflammation in HFD-fed mice. Moreover, RD supplementation reduces macrophage infiltration, regulates polarization of macrophage and inhibits the NF-kB signaling pathway in epididymal adipose tissue. Our study demonstrates that RD may represent a new non-drug therapy to prevent and treat obesity.

\section{Materials and Methods}

\section{Animals and sample collection}

Male C57BL/6 mice, aged four weeks, were purchased from the Shanghai SLAC Laboratory Animal Co. Ltd. Four animals were housed per cage with free access to food and sterile drinking water in a climate-controlled room $\left(21^{\circ} \mathrm{C} \pm 2^{\circ} \mathrm{C}\right)$ under a 12-hour light/dark cycle (7:00 AM-7:00 PM). After a week of acclimatization, mice were randomly divided into three groups: control chow group (chow), high-fat diet group (HFD) and high-fat diet supplemented with resistant dextrin group (HFD+RD), and each group consisted of 12 mice. The chow group mice were fed for 12 weeks with a standard diet, and the other two groups were fed with a HFD (45\% energy from fat; D12451, Research Diet, USA). In addition, the HFD+RD group mice were orally administered 10 $\mathrm{g} / \mathrm{kg} \mathrm{RD}$, and the other two groups were orally administered equal sterile saline once per day for 12 weeks. All procedures were performed in accordance with the National Institutes of Health Guidelines for the Care and Use of Animals and were approved by the Ethics Committee of Xinhua Hospital affiliated with Shanghai Jiao Tong University School of Medicine.

Mice were fasted overnight for $16 \mathrm{~h}$ then anesthetized, and whole blood samples were collected via eyeball extraction. Epididymal white adipose tissue was sampled and weighed. Tissue samples were immediately placed in liquid nitrogen and stored at $-80^{\circ} \mathrm{C}$ before the assay.

\section{Reagents and antibodies}

RD was purchased from Guangzhou Honsea Industry Co. Ltd. Rabbit monoclonal antibodies against phospho-Ser32 IкBa (\#2859), p65 (\#8242), phospho-Ser536 p65 (\#3033), $\beta$-actin (\#4970) and a mouse monoclonal antibody against IkBa (\#4814) were purchased from Cell Signaling Technology (Cell Signaling, USA). Goat polyclonal secondary antibodies against mouse (\#A0216) or rabbit (\#A0208) were purchased from Beyotime Biotechnology (Beyotime, China).

\section{Histological analysis and immunohistochemical staining}

Epididymal white adipose tissue samples from all groups of mice were fixed in phosphate-buffered $10 \%$ formalin and embedded in paraffin wax. On one hand, sections were cut ( $4 \mu \mathrm{m}$ thick) and stained with hematoxylin and eosin (H\&E). The quantification of adipocyte size was performed in five microscopy fields for each mouse using ImageJ software (magnification 200x). On the other hand, the slices were dewaxed with xylene and rehydrated with graded ethanol to water. The slices were then placed in citric acid antigen repair buffer and heated in a microwave oven for antigen repair. Slices were incubated with $3 \% \quad \mathrm{H}_{2} \mathrm{O}_{2}$ in the dark at room temperature for 25 minutes to block the activity of endogenous peroxidase. 3\% BSA was used for background blocking for 30 minutes. The slices were incubated with primary rabbit anti-mouse F4/80 at $4^{\circ} \mathrm{C}$ overnight. After washing the slices three times, slices were incubated with HRP-conjugated secondary antibody for $50 \mathrm{~min}$. After washing the slices another three times, color was developed using the diaminobenzidine substrate and counterstained with hematoxylin. Microscope was used to collect pictures, then ImageJ was used to calculate the percentage of F4/80-positive staining area per slice [23].

\section{Serum total triglyceride measurements}

Blood samples were centrifuged, and plasma was collected and stored at $-80^{\circ} \mathrm{C}$ before the assay. Total triglyceride (TG) levels of all the samples were 
tested in an automatic biochemical analyzer (Hitachi, Japan).

\section{Serum free fatty acid measurements}

Serum free fatty acid (FFA) levels were measured using commercial kits (Nanjing Jiancheng Bioengineering Institute, China).

\section{Enzyme-linked immunosorbent assay (ELISA)}

Serum lipopolysaccharide (LPS), tumor necrosis factor-alpha (TNF- $\alpha)$ interleukin-1 $\beta$ (IL-1 $\beta)$, interleukin-6 (IL-6) and interleukin-10 (IL-10) levels were quantified according to the protocol of the corresponding ELISA kit (Westang, China).

\section{Quantitative real-time polymerase chain reaction (PCR)}

Total RNA was extracted from frozen tissues using Trizol reagent according to the manufacturer's instructions (Takara, Japan). Then, the total RNA was converted to complementary deoxyribonucleic acid (cDNA) using a reverse transcription reagent kit (Takara, Japan). Quantitative real-time PCR was performed with SYBR Green PCR reagent (Takara, Japan) for quantification of the mRNA levels. Calculations were made based on the comparative cycle threshold method $\left(2^{-{ }^{\Delta A} C t}\right)$. The chow group was used as calibrator group and glyceraldehyde 3-phosphate dehydrogenase (GAPDH) was used as an endogenous normalization control. Primer sequences are given in Table 1.

\section{Western blot analysis}

RIPA lysis buffer containing protease and phosphatase inhibitor cocktails was used to extract proteins. Then, the extracted proteins were quantified with a BCA Protein Quantitative Analysis kit (Beyotime, China). A total of $20 \mu \mathrm{g}$ of the protein sample was separated on an SDS PAGE gel and transferred to a polyvinylidene fluoride (PVDF) membrane (Millipore, USA). After that, the membrane was blocked with $5 \%$ skim milk powder and incubated overnight with primary antibodies. Next, the membrane was washed three times and incubated with an HRP-conjugated secondary antibody. Finally, the membrane was washed another three times, and the protein bands were detected with Immobilon Western Chemiluminescent HRP Substrate (Millipore, USA). ImageJ software was used to quantify the protein bands.

\section{Statistical analysis}

All data are shown as the mean \pm standard error of the mean (SEM). All statistical analyses were performed using GraphPad Prism V.7.0. One-way or two-way analysis of variance (ANOVA) followed by Bonferroni's post hoc test was used for comparison of multiple groups. A two-tailed unpaired $\mathrm{t}$ test was used for comparison of two groups. A value of $p<$ 0.05 was considered statistically significant.

\section{Results}

\section{RD reduced HFD-induced obesity in mice}

To study the effects of RD on obesity, the obesity model induced by HFD was used in this study. After 12 weeks of HFD feeding, the BW, body weight gain, FBG, epididymal fat accumulation and adipocyte size were increased significantly compared with control chow group (Fig. 1A-F). However, RD treatment reduced the $\mathrm{BW}$, body weight gain, FBG, epididymal fat accumulation and adipocyte size compared with HFD group (Fig. 1A-F). In addition, RD supplementation increased food intake compared with HFD group (Fig. 1G). Adipose tissue can store nutrients in the form of triglycerides. To investigate whether RD alleviates the serum lipid level, a quantitative analysis of serum TG and FFA levels was performed. We observed that RD supplementation reduced serum TG and FFA levels in HFD-fed mice (Fig. 1H \& I). These results indicate that RD prevents HFD-induced obesity in mice.

Table 1. Sequence of primers for quantitative real-time PCR

\begin{tabular}{|c|c|c|c|}
\hline Gene & Species & Forward primer & Reverse primer \\
\hline TNF-a & Mouse & ATGTCTCAGCCTCTTCTCATTC & GCTTGTCACTCGAATTTTGAGA \\
\hline IL-1 $\beta$ & Mouse & TCGCAGCAGCACATCAACAAGAG & AGGTCCACGGGAAAGACACAGG \\
\hline IL-6 & Mouse & CTGCAAGAGACTTCCATCCAG & AGTGGTATAGACAGGTCTGTTGG \\
\hline IL-10 & Mouse & TTCTTTCAAACAAAGGACCAGC & GCAACССАAGTAACССТTAAAG \\
\hline $\mathrm{F} 4 / 80$ & Mouse & TGTCTGCATGATCATCACGATA & CGTGTCCTTGAGTTTAGAGACT \\
\hline MCP1 & Mouse & TTTTTGTCACCAAGCTCAAGAG & TTCTGATCTCATTTGGTTCCGA \\
\hline Nos2 & Mouse & AGGCCACATCGGATTTCACT & TCAATGGCATGAGGCAGGAG \\
\hline TLR4 & Mouse & GCCATCATTATGAGTGCCAATT & AGGGATAAGAACGCTGAGAATT \\
\hline Arg1 & Mouse & AGACCACAGTCTGGCAGTTGG & AGGTTGCCCATGCAGATTCCC \\
\hline PPAR Y & Mouse & CCAAGAATACCAAAGTGCGATC & TCACAAGCATGAACTCCATAGT \\
\hline P65 & Mouse & ATTTCCGCCTCTGGCGAATG & GATGAGGGGAAACAGATCGTCC \\
\hline GAPDH & Mouse & AAGAAGGTGGTGAAGCAGGCATC & CGGCATCGAAGGTGGAAGAGTG \\
\hline
\end{tabular}


A

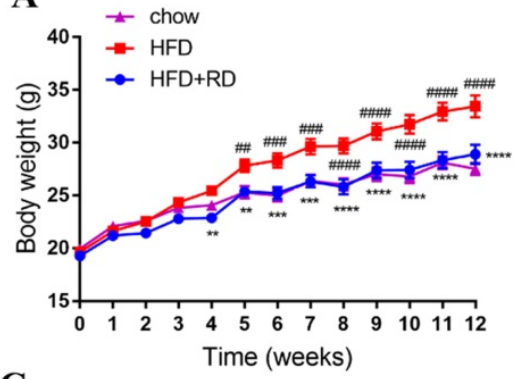

C

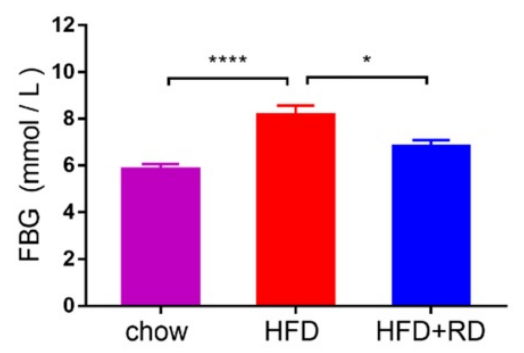

E

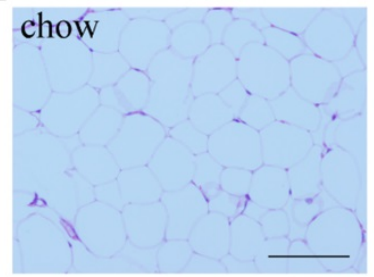

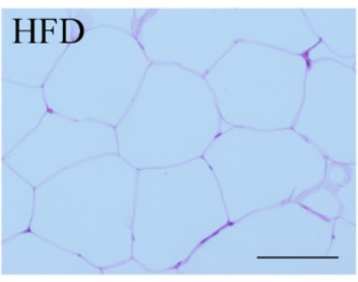

B

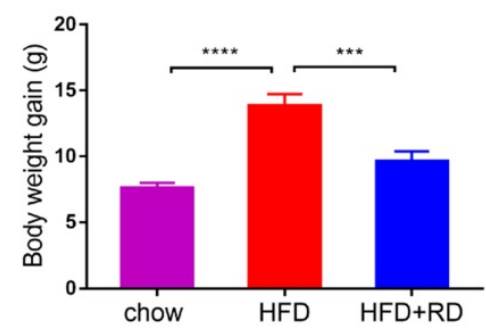

D

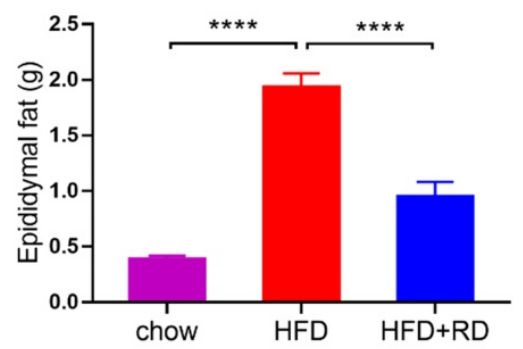

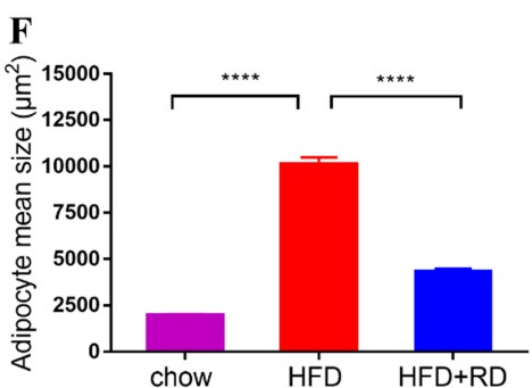

H

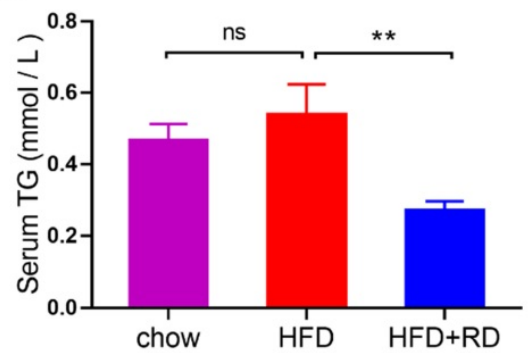

G

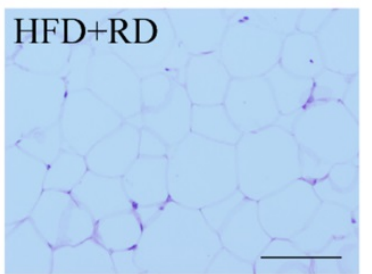

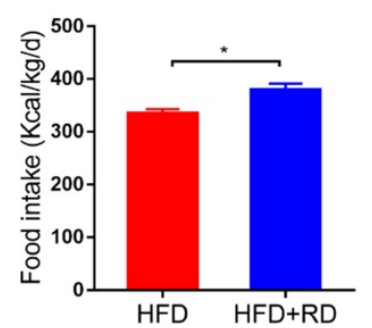

I

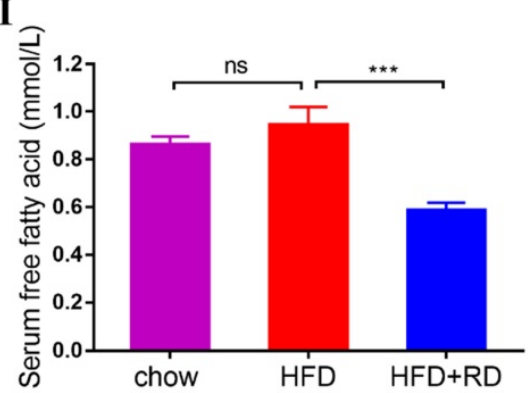

Figure 1. RD reduces HFD-induced obesity in mice. Chow- and HFD-fed mice were treated daily with sterile saline or RD by intragastric gavage for 12 weeks. (A) Body weight. (B) Body weight gain at week 12. (C) FBG at week 12. (D) Epididymal fat accumulation. (E) Representative images of hematoxylin and eosin (H\&E)-stained epididymal adipose tissues. Scale bar, $100 \mu \mathrm{m}$; magnification, $\times 200$. (F) Adipocyte size in epididymal adipose tissues was determined using Image J software. (G) Food intake at week 12 . (H) Serum TG. (I) Serum free fatty acid. Data are presented as mean \pm SEM, $n=12$ mice per group. Statistical analysis was performed using two-way (panel $A$ ) or one-way (Except

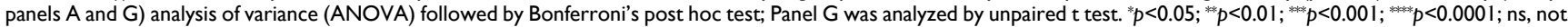
statistically significant. In panel A, ${ }^{* *} p<0.01 ;{ }^{* * *} p<0.001$; ${ }^{* * * *} p<0.0001$ versus HFD; \#\# $p<0.01$; \#\# $p<0.001$; \#\#\#\# $p<0.0001$ versus chow.

\section{RD reduces proinflammatory cytokine levels in HFD-fed mice}

Obesity-associated chronic tissue inflammation is a key contributory factor to type 2 diabetes and cardiovascular disease, and previous studies have clearly suggested that metabolism and immune system are closely integrated [24]. To determine 
whether RD regulates inflammatory cytokine levels, we performed a quantitative analysis of the serum LPS, TNF- $\alpha$, IL-1 $\beta$, IL-6, and IL-10 levels. We observed that LPS and IL-6 levels were higher in HFD-fed mice compared with chow-fed mice, and serum IL-6 level was significantly reduced in the RD supplementation group compared with the HFD group (Fig. 2A \& D). Moreover, HFD reduced the serum level of the anti-inflammatory cytokine IL-10, which was slightly increased in RD supplementation group (Fig. 2E). Next, we examined the mRNA expression levels of proinflammatory genes and anti-inflammatory genes, including TNF- $\alpha$, IL-1 $\beta$, IL-6, and IL-10, in epididymal adipose tissue. Similar to the serum inflammatory cytokine result, the mRNA level of IL-6 was reduced by RD supplementation (Fig. 3C). However, no significant alteration was found in the mRNA expression levels of TNF- $\alpha$, IL-1 $\beta$, and IL-10 (Fig. 3A, $B \quad \& \quad D)$. These results indicate that $R D$ supplementation reduces proinflammatory cytokine expression and production in HFD-fed mice.

A

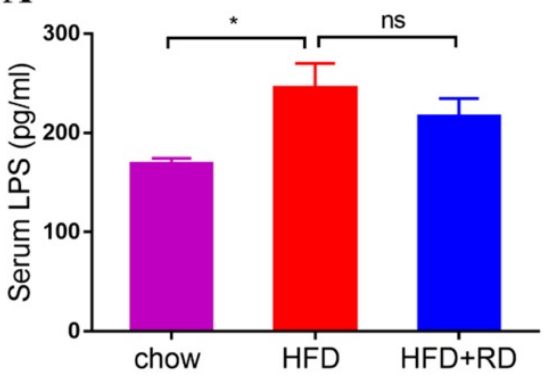

C

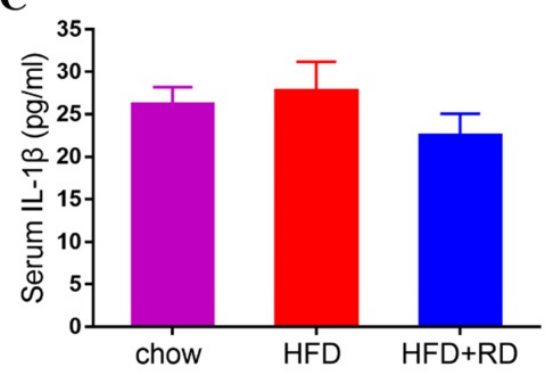

E

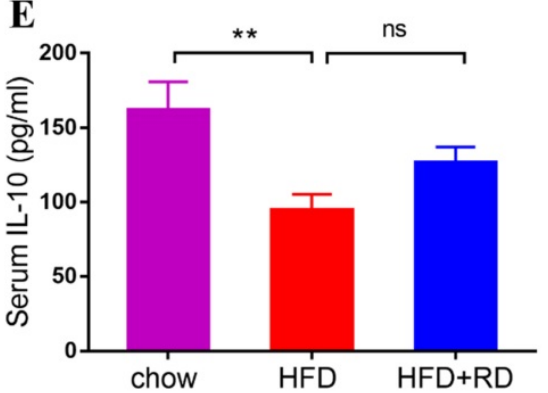

RD supplementation reduces macrophage infiltration in epididymal adipose tissue of HFD-fed mice

Obesity is characterized by infiltration and activation of macrophages in adipose tissues, which results chronic low-grade inflammation [25]. Therefore, the quantification of F4/80-positive macrophages was observed by immunohistochemical staining in epididymal white adipose tissue. The results showed that the percentage of F4/80-positive staining area was increased in HFD group compared with chow group and RD supplementation significantly reversed macrophage infiltration (Fig. $4 \mathrm{~A} \& \mathrm{~B})$. In addition, the mRNA level of F4/80 was also significantly reduced in RD supplementation group compared with HFD-fed mice (Fig. 4C). The mRNA levels of proinflammatory cytokines monocyte chemoattractant protein 1 (MCP1) and M1 macrophage marker nitric oxide synthase 2 (Nos2) were all increased in HFD-fed mice compared with chow-fed mice. RD supplementation significantly

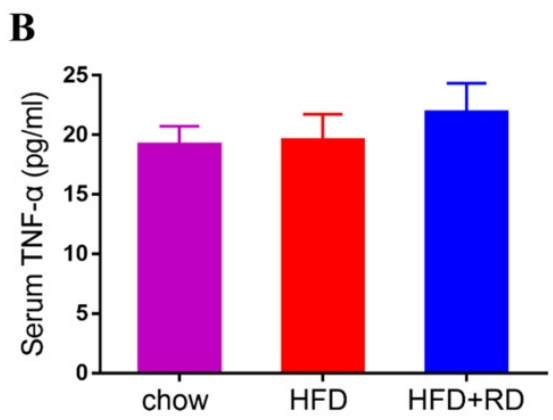

D

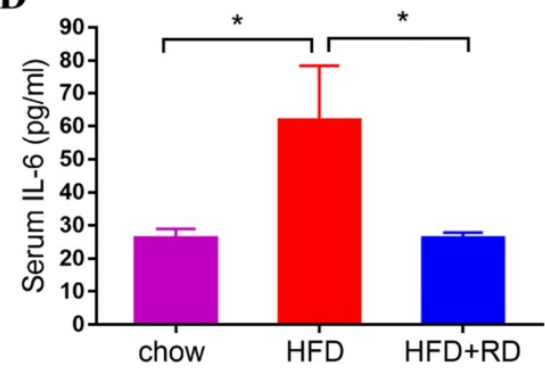

Figure 2. RD reduces proinflammatory cytokine levels in HFD-fed mice. (A-E) Serum LPS (A), TNF- $\alpha$ (B), IL-1 13 (C), IL-6 (D), IL-10 (E) levels were determined using ELISA at week 12. N=12 mice per group. Data are presented as mean \pm SEM. Statistical analysis was performed using one-way analysis of variance (ANOVA) followed by Bonferroni's post hoc test. ${ }^{*} p<0.05 ;{ }^{* *} p<0.01 ; * * * * 0.001 ;{ }^{* * * * * *} p<0.0001 ;$ ns, not statistically significant. 
reversed MCP1 and Nos2 gene expression levels in HFD-fed mice (Fig. 4D-E). Moreover, the mRNA level of M2 macrophage marker peroxisome proliferatorsactivated receptor $\gamma(P P A R \gamma)$ was reduced in HFD-fed mice compared with chow group. RD supplementation significantly increased PPARY expression level and also tended to increase Arg1 level compared with HFD group (Fig. 4F-G). These results indicate that $\mathrm{RD}$ supplementation reduces macrophage infiltration and regulates polarization of macrophage in epididymal adipose tissue of HFD-fed mice.

\section{RD inhibits NF-KB signaling pathways in the adipose tissue of HFD-fed mice}

Previous studies have indicated that FFA and LPS might promote adipose tissue inflammation by binding to Toll-like receptors (TLRs), such as TLR4 [26]. TLR4 activates downstream NF- $\mathrm{KB}$ signaling to increases the synthesis and secretion of MCP1, which promotes macrophage recruitment to adipose tissue and the development of insulin resistance [27-29]. Therefore, we further explored the mRNA expression level of TLR4. The results showed that the mRNA level of TLR4 in the epididymal white adipose tissue of RD-treated mice was lower than that of HFD-fed mice (Fig. 5A). Moreover, we examined whether the NF-kB signaling pathway was affected by RD supplementation in epididymal white adipose tissue. The protein expression level of $\mathrm{I} \kappa \mathrm{Ba}$, which is an inhibitor of the transcription factor NF- $\mathrm{kB}$, was upregulated in RD-treated mice compared with

A

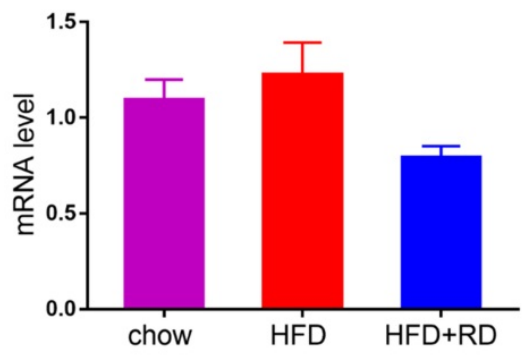

C

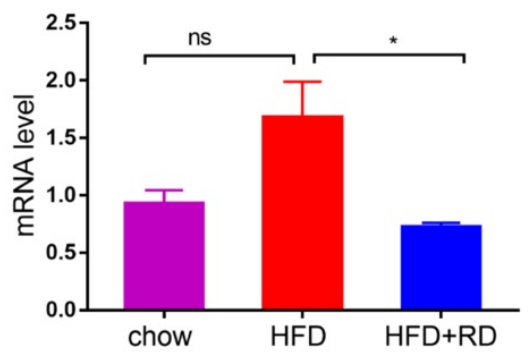

HFD-fed mice. In contrast, the level of phospho-Ser32 IкBa, which leads to the release of active NF-кB and nuclear translocation, was reduced in RDsupplemented mice compared with HFD-fed mice (Fig. 5C). RD supplementation reduced phosphoSer536 p65 expression level compared with HFD group (Fig. 5C). These results indicate that $\mathrm{RD}$ supplementation inhibits NF- $\mathrm{kB}$ signaling in epididymal adipose tissue of HFD-fed mice.

\section{Discussion}

Several randomized clinical trials have shown that RD improves insulin resistance, glycemic control, and blood pressure and modulates inflammation [16, $17,30,31]$. A previous study shown that body weight was reduced in overweight men who were given $17 \mathrm{~g}$ RD supplementation twice daily [22]. However, the improvement of adipose tissue inflammation and specific mechanism of RD in HFD-induced obesity were not fully understood. In the present study, we mainly studied the effects of RD on epididymal fat, which is the typical representative of visceral fat and closely associated with obesity-related diseases. We found that RD reduced obesity and attenuated adipose tissue inflammation in HFD-fed mice, including reducing body weight gain, FBG, epididymal fat accumulation, adipocyte size, proinflammatory cytokines and macrophage infiltration. Our research suggests that RD may represent a new non-drug therapy to prevent and treat obesity.

\section{B}

IL-1 $\beta$

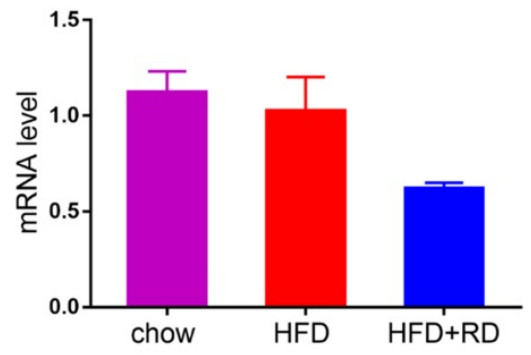

D

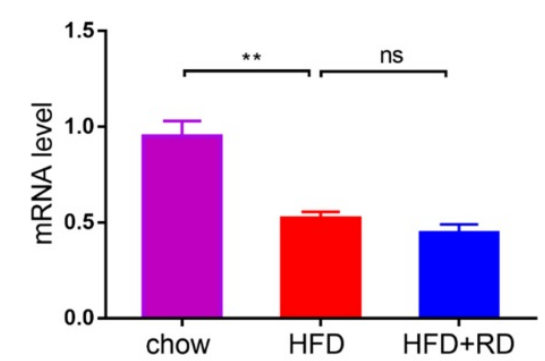

Figure 3. RD reduces the expression levels of proinflammatory genes in HFD-fed mice. (A-C) qPCR analysis of proinflammatory related genes TNF- $\alpha$ ( $A$ ), IL-1 $\beta$ (B), and IL-6 (C) levels in epididymal adipose tissue. $N=4$ mice per group. (D) qPCR analysis of anti-inflammatory gene IL-10 level in epididymal adipose tissue. $N=4$ mice per group. Data are presented as mean \pm SEM. Statistical analysis was performed using one-way analysis of variance (ANOVA) followed by Bonferroni's post hoc test. ${ }^{*} p<0.05$; ${ }^{* *}<0.01$; **** $p<0.001 ; * * * 0.0001 ;$ ns, not statistically significant. 
A
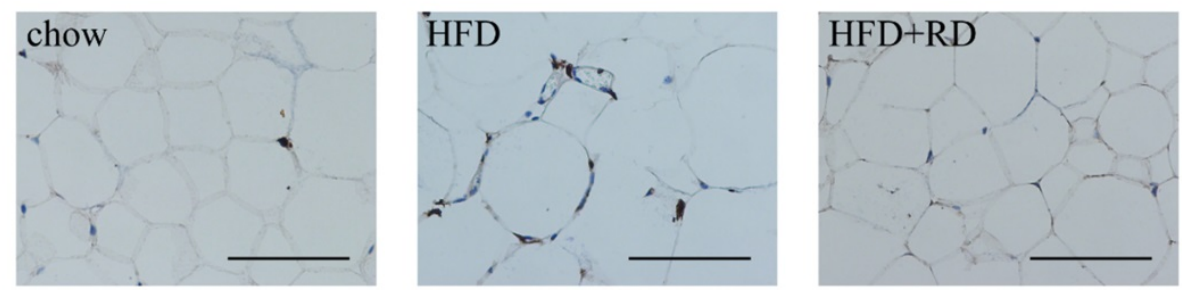

B

C

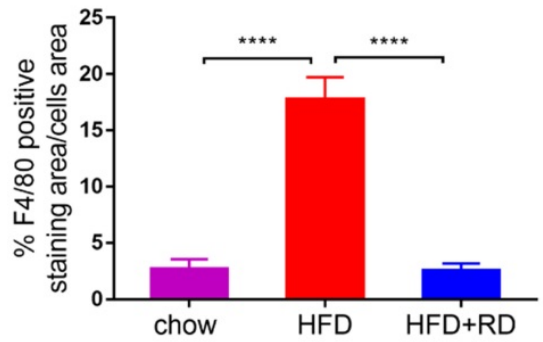

$\mathrm{F} 4 / 80$

D

MCP1

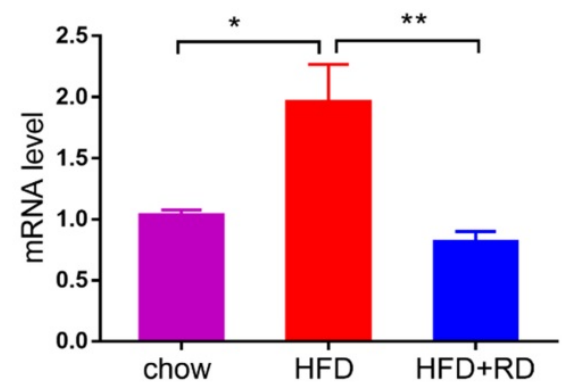

$\mathbf{E}$

Nos2

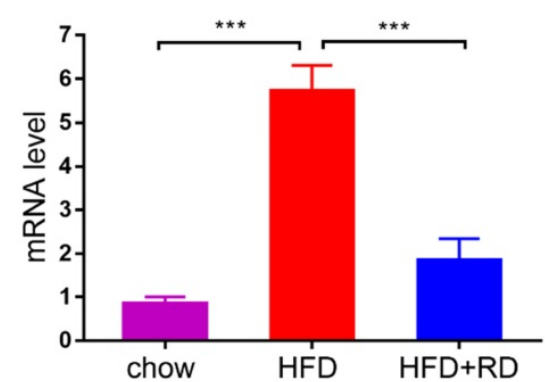

F

Arg1

G
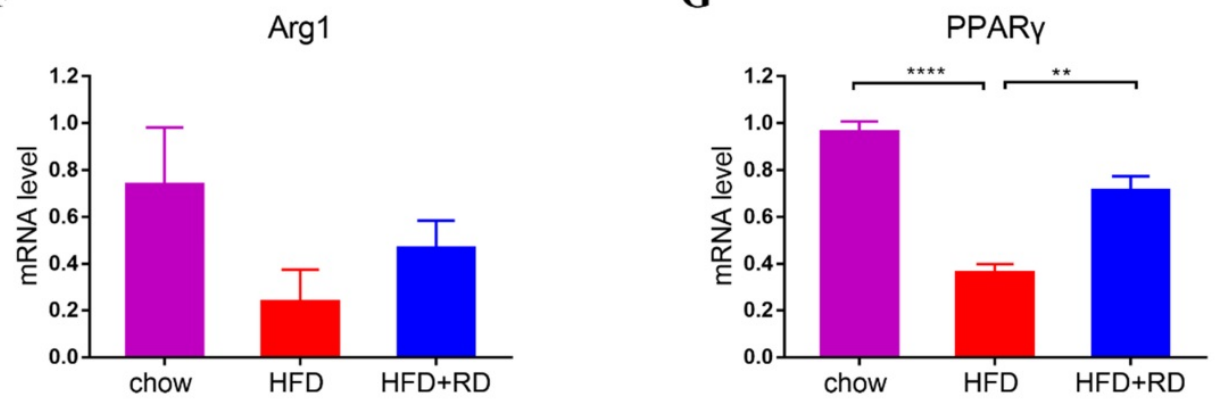

Figures 4. RD supplementation reduces macrophage infiltration in epididymal adipose tissue of HFD-fed mice. (A) Representative images of immunohistochemical stain of epididymal adipose tissue against the specific macrophage maker F4/80. Scale bar, $100 \mu$ m; magnification, $\times 200$. (B) Quantification of F4/80-positive staining area in epididymal adipose tissue. $N=6$ mice per group. (C-G) qPCR analysis of the mRNA level of F4/80 (C), MCPI (D), Nos2 (E), Arg1 (F) and PPAR $\gamma$ (G) in epididymal adipose tissue. $\mathrm{N}=4$ mice per group. Data are presented as mean \pm SEM. Statistical analysis was performed using one-way analysis of variance (ANOVA) followed by Bonferroni's post hoc test. ${ }^{*} p<0.05 ;{ }^{* * *} p<0.01 ; * * * 0<0.001 ;{ }^{* * * * * *} p<0.0001$; ns, not statistically significant.

Many studies have indicated that HFD can induce adipocyte expansion in size and number, which aims to accommodate the need for excessive TG storage and the anabolic force of hyperinsulinemia [32-34]. Our study shown that RD reduced visceral fat accumulation, adipocyte hypertrophy and serum TG. When adipocytes reach a certain metabolic stress, an inflammatory program is to be triggered. The triggers of the inflammatory program may originate from a gut-derived substance, dietary component and metabolite [26]. Previous studies have shown that HFD can disrupt gut barrier integrity and lead to translocation of LPS from gram-negative bacteria into the blood, which produces inflammation and insulin resistance $[35,36]$. Moreover, elevated concentration of free fatty acid in HFD-fed mice can also contribute to adipose tissue inflammation. High circulating levels of LPS and FFA can cause systemic and 
targeted inflammation in HFD-fed mice by activating TLR4 signaling [37-39]. A previous study has shown that TLR4 $\%$ HFD-fed mice significantly reduces adipose tissue inflammatory markers and macrophage recruitment [40]. We found that RD reduced the serum levels of FFA and LPS, and the mRNA level of TLR4 was also reduced in epididymal adipose tissue.

HFD activates several proinflammatory signaling pathways, and NF- $\mathrm{kB}$ signaling is a crucial pathway in this process, which is the downstream of TLR4 and leads to the development of insulin resistance, cytokine production, and eventually immune cell recruitment [41]. A previous study has shown that inhibition of inflammatory signaling by knockout of NF-kB pathway can disrupt the link between obesity and insulin resistance in obese mice [42]. Another study shown that stevioside (SVS), a widely used sweetener with multiple beneficial effects for diabetic patients, reduced expression levels of TNF- $\alpha$, IL-6, IL-1 $\beta$, macrophage inflammatory protein-1a (MIP-1a), CD11b and CD14 and inhibited the NF-KB signaling pathway in adipose tissue [43]. Moreover, a water extract of Ganoderma lucidum mycelium (WEGL) reduced endotoxemia and enhanced the expression of IKBa in adipose tissue in HFD mice [32]. In the present study, we found that $\mathrm{RD}$ increased the protein expression level of IкBa, which interacts with the transcription factor NF- $\mathrm{KB}$ and prevents NF- $\mathrm{kB}$ translocation and activation. In addition, the protein level of $\mathrm{p}$-ІкBa was reduced in RD-supplemented mice compared with HFD-fed mice. Moreover, RD supplementation reduced the p-p65 expression levels compared with HFD group. Activation of NF-kB signaling pathway can increase the synthesis and secretion of MCP1 in adipocytes. MCP1 can promote macrophage recruitment to adipose tissue and the development of insulin resistance. Our results showed that the mRNA level of MCP1 was upregulated significantly in the HFD-fed mice, which was reversed by RD supplementation.
A

A

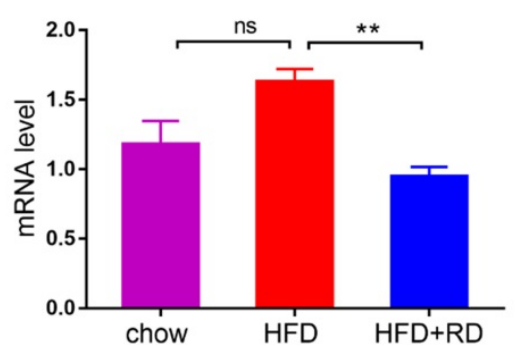

B

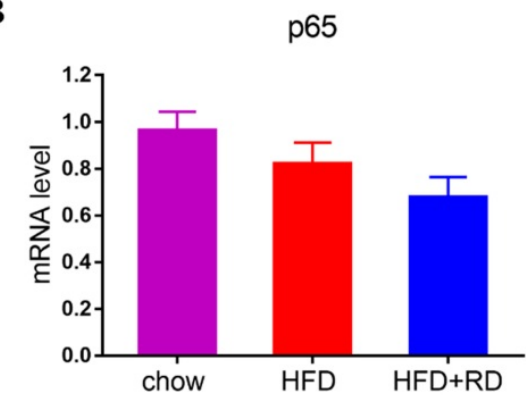

C

Epididymal adipose
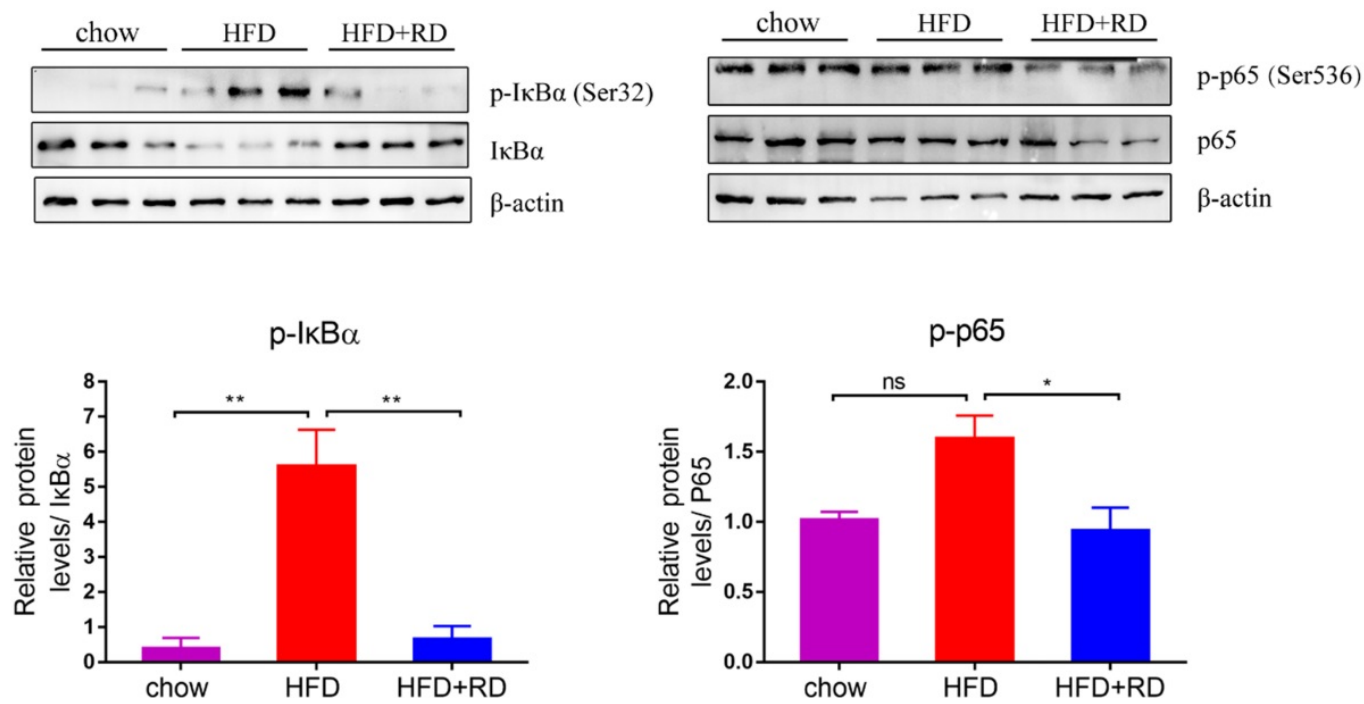

Figure 5. RD inhibits NF-kB signaling pathways in epididymal adipose tissue of HFD-fed mice. (A-B) qPCR analysis of the mRNA level of TLR4 (A) and p65 (B) in epididymal adipose tissue. $\mathrm{N}=4$ mice per group. (C) Western blot analysis of NF-KB signaling pathway related proteins expression levels in epididymal adipose tissue. $\mathrm{N}=3$ mice per group. Data are presented as mean \pm SEM. Statistical analysis was performed using one-way analysis of variance (ANOVA) followed by Bonferroni's post hoc test. ${ }^{*}<<0.05$;

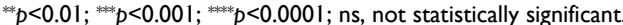


Many studies have demonstrated that obesity is closely associated with an overall increase of macrophage infiltration in both rodents and humans [44]. Macrophages are generally grouped as M1 and M2 phenotypes, which respectively represent pro- or anti-inflammatory phenotypes. The expression of maker genes for M1 and M2 macrophages from mouse epididymal fat tissue are different. TNF- $\alpha$, IL-1 $\beta$, IL-6 and Nos2 were highly expressed in the M1 macrophages, and CD206, CD163, IL-10, Arg1, PPARY, Mrc1 and Fizz1 were highly expressed in the M2 macrophages [45, 46]. The macrophages that appear in large numbers in the adipose tissue of obese people are often referred to as M1 macrophages [47]. On the one hand, pro-inflammatory macrophages are recruited from blood monocytes to adipose tissue [48], on the other hand, resident classically activated macrophages surround the dead adipocytes and form crown-like structures (CLS) [49]. In the present study, we found that RD reduced macrophage infiltration and regulated polarization of macrophage towards the M2 type in epididymal adipose tissue of HFD-fed mice, which reduced IL-6 and Nos 2 expression levels and increased PPARY level.

The gut microbiota of obese human and mice is associated with decreased level of intestinal Bacteroidetes and increased level of Firmicutes [50, 51]. Many studies have been performed to investigate the effect of dietary fiber on the modulation of gut microbiota. Dietary fibers can alleviate type 2 diabetes by promoting a specific group of short-chain fatty acid (SCFA)-producing gut bacteria [52]. Inulin supplementation alleviates glucose and lipid metabolism disorders by modulating the gut microbiota in ob/ob mice [53]. The major metabolites from the microbial fermentative activity in the gut are SCFAs, including acetate, propionate, and butyrate. GPR41, GPR43 and GPR109A are considered SCFAs receptors [54, 55]. A study showed that the GPR109A agonist butyrate reduced LPS-induced NF-кB activation in mouse colons and KM12L4 cancer cell line [56]. GPR43 plays an important role in white adipose tissue. Adipose-specific overexpression of GPR43 mice can remain lean, even when the mice were fed a HFD. However, the effects were abrogated when mice were raised under germ-free conditions [57]. Moreover, a previous study has indicated that RD from maize starch induces the growth of Bacteroidetes and Actinobacteria and inhibits the growth of Firmicutes in obesity [58]. Another study has shown that RD contributes to the growth of butyrate- and acetate-producing microbes in male IL-10-/-mice [59]. Therefore, the modulation of gut microbiota may also play an important role in metabolically beneficial effects of RD supplementation mice.

\section{Conclusions}

In summary, RD supplementation reduces body weight gain, FBG, visceral fat accumulation, adipocyte hypertrophy, proinflammatory cytokines, macrophages infiltration and regulates polarization of macrophage in epididymal adipose tissue of HFD-fed mice. Moreover, RD supplementation inhibits NF- $\mathrm{kB}$ signaling in epididymal adipose tissue, which may be a presumed mechanism for metabolically beneficial effects. Our research suggests that RD may represent a new non-drug therapy to prevent and treat the major global problem obesity.

\section{Acknowledgments}

We thank Xinhua Hospital Shanghai Jiao Tong University School of Medicine for supporting this study.

\section{Funding}

This work was supported by the grants 2016YFC0901200 and 2016YFC0901203 from the Ministry of Science and Technology, national Natural Science Foundation of China (No. 81670743), Shanghai Sailing Program (18YF1415800) and Clinical Research Plan of SHDC (16CR4012A).

\section{Authors' Contributions}

Q.S., X.L., Q.H. and F.H. conceived and designed the experiments; Q.H., Y.L., S.H. and X.X. performed the experiments and analyzed the data; Q.H. and F.H. drafted the manuscript; Q.H., Y.L., Q.S., X.L., Y.N., and H.Z. reviewed and revised the manuscript; all authors approved the final version of the manuscript.

\section{Competing Interests}

The authors have declared that no competing interest exists.

\section{References}

1. Stefan N, Häring $\mathrm{H}-\mathrm{U}, \mathrm{Hu}$ FB, Schulze MB. Metabolically healthy obesity: epidemiology, mechanisms, and clinical implications. Lancet Diabetes Endocrinol. 2013;1(2):152-62.

2. Wilding JPH, Mooney V, Pile R. Should obesity be recognised as a disease? BMJ. 2019;366:14258.

3. Maner JK, Dittmann A, Meltzer AL, McNulty JK. Implications of life-history strategies for obesity. Proc Natl Acad Sci U S A. 2017;114(32):8517-22.

4. Borgland SL. Releasing the brake on eating. Science. 2019;364(6447):1233-4.

5. Wolfe BM, Kvach E, Eckel RH. Treatment of Obesity. Circ Res. 2016;118(11):1844-55.

6. Kivimaki M, Jokela M, Hamer M, Geddes J, Ebmeier K, Kumari M, et al. Examining overweight and obesity as risk factors for common mental disorders using fat mass and obesity-associated (FTO) genotype-instrumented analysis: The Whitehall II Study, 1985-2004. Am J Epidemiol. 2011;173(4):421-9.

7. Ahima RS. Digging deeper into obesity. J Clin Invest. 2011;121(6):2076-9. 
8. Livingston EH, Zylke JW. Progress in obesity research: reasons for optimism. JAMA. 2012;308(11):1162-4.

9. Poirier P, Cornier MA, Mazzone T, Stiles S, Cummings S, Klein S, et al. Bariatric surgery and cardiovascular risk factors: a scientific statement from the American Heart Association. Circulation. 2011;123(15):1683-701.

10. Dixon JB, Schachter LM, O'Brien PE, Jones K, Grima M, Lambert G, et al. Surgical vs conventional therapy for weight loss treatment of obstructive sleep apnea: a randomized controlled trial. JAMA. 2012;308(11):1142-9.

11. Trends in adult body-mass index in 200 countries from 1975 to 2014: a pooled analysis of 1698 population-based measurement studies with 19.2 million participants. Lancet. 2016;387(10026):1377-96.

12. Holscher HD. Dietary fiber and prebiotics and the gastrointestinal microbiota. Gut Microbes. 2017;8(2):172-84.

13. Li S, Flint A, Pai JK, Forman JP, Hu FB, Willett WC, et al. Dietary fiber intake and mortality among survivors of myocardial infarction: prospective cohort study. BMJ. 2014;348:g2659.

14. Trithavisup K, Krusong K, Tananuwong K. In-depth study of the changes in properties and molecular structure of cassava starch during resistant dextrin preparation. Food Chem. 2019;297:124996.

15. Farhangi MA, Javid AZ, Sarmadi B, Karimi P, Dehghan P. A randomized controlled trial on the efficacy of resistant dextrin, as functional food, in women with type 2 diabetes: Targeting the hypothalamic-pituitary-adrenal axis and immune system. Clin Nutr. 2018;37(4):1216-23.

16. Mateo-Gallego R, Pérez-Calahorra S, Lamiquiz-Moneo I, et al. Effect of an alcohol-free beer enriched with isomaltulose and a resistant dextrin on insulin resistance in diabetic patients with overweight or obesity. Clin Nutr. 2020;39(2):475-83.

17. Cai X, Yu H, Liu L, Lu T, Li J, Ji Y, et al. Milk Powder Co-Supplemented with Inulin and Resistant Dextrin Improves Glycemic Control and Insulin Resistance in Elderly Type 2 Diabetes Mellitus: A 12-Week Randomized, Double-Blind, Placebo-Controlled Trial. Mol Nutr Food Res. 2018;62(24):e1800865.

18. Gholizadeh Shamasbi S, Dehgan P, Mohammad-Alizadeh Charandabi S, Aliasgarzadeh A, Mirghafourvand $\mathrm{M}$. The effect of resistant dextrin as a prebiotic on metabolic parameters and androgen level in women with polycystic ovarian syndrome: a randomized, triple-blind, controlled, clinical trial. Eur J Nutr. 2019;58(2):629-40.

19. van den Heuvel EG, Wils D, Pasman WJ, Bakker M, Saniez MH, Kardinaal AF. Short-term digestive tolerance of different doses of NUTRIOSE FB, a food dextrin, in adult men. Eur J Nutr. 2004;58(7):1046-55.

20. Pasman W, Wils D, Saniez MH, Kardinaal A. Long-term gastrointestinal tolerance of NUTRIOSE FB in healthy men. Eur J Nutr. 2006;60(8):1024-34.

21. Vermorel M, Coudray C, Wils D, Sinaud S, Tressol JC, Montaurier C, et al. Energy value of a low-digestible carbohydrate, NUTRIOSE FB, and its impact on magnesium, calcium and zinc apparent absorption and retention in healthy young men. Eur J Nutr. 2004;43(6):344-52.

22. Guerin-Deremaux L, Li S, Pochat M, Wils D, Mubasher M, Reifer C, et al. Effects of NUTRIOSE(R) dietary fiber supplementation on body weight, body composition, energy intake, and hunger in overweight men. Int J Food Sci Nutr. 2011;62(6):628-35.

23. Martínez-Fernández L, González-Muniesa P, Laiglesia LM, et al. Maresin 1 improves insulin sensitivity and attenuates adipose tissue inflammation in ob/ob and diet-induced obese mice. FASEB J. 2017;31(5):2135-2145

24. Osborn $\mathrm{O}$, Olefsky JM. The cellular and signaling networks linking the immune system and metabolism in disease. Nat Med. 2012;18(3):363-74.

25. Ferrante AW, Jr. Obesity-induced inflammation: a metabolic dialogue in the language of inflammation. J Intern Med. 2007;262(4):408-14.

26. Reilly SM, Saltiel AR. Adapting to obesity with adipose tissue inflammation. Nat Rev Endocrinol. 2017;13(11):633-43.

27. Neels JG, Olefsky JM. Inflamed fat: what starts the fire? J Clin Invest. 2006;116(1):33-5.

28. Weisberg SP, Hunter D, Huber R, Lemieux J, Slaymaker S, Vaddi K, et al. CCR2 modulates inflammatory and metabolic effects of high-fat feeding. J Clin Invest. 2006;116(1):115-24.

29. Kamei N, Tobe K, Suzuki R, Ohsugi M, Watanabe T, Kubota N, et al. Overexpression of monocyte chemoattractant protein-1 in adipose tissues causes macrophage recruitment and insulin resistance. J Biol Chem. 2006;281(36):26602-14

30. Li S, Guerin-Deremaux L, Pochat M, Wils D, Reifer C, Miller LE. NUTRIOSE dietary fiber supplementation improves insulin resistance and determinants of metabolic syndrome in overweight men: a double-blind, randomized, placebo-controlled study. Appl Physiol Nutr Metab. 2010;35(6):773-82
31. Aliasgharzadeh A, Dehghan P, Gargari BP, Asghari-Jafarabadi M. Resistant dextrin, as a prebiotic, improves insulin resistance and inflammation in women with type 2 diabetes: a randomised controlled clinical trial. Br J Nutr. 2015;113(2):321-30.

32. Chang CJ, Lin CS, Lu CC, Martel J, Ko YF, Ojcius DM, et al. Ganoderma lucidum reduces obesity in mice by modulating the composition of the gut microbiota. Nat Commun. 2015;6:7489.

33. Li J, Gong L, Liu S, Zhang Y, Zhang C, Tian M, et al. Adipose HuR protects against diet-induced obesity and insulin resistance. Nat Commun. 2019;10(1):2375.

34. Rosen ED, Spiegelman BM. What we talk about when we talk about fat. Cell. 2014;156(1-2):20-44.

35. Cani PD, Bibiloni R, Knauf C, Waget A, Neyrinck AM, Delzenne NM, et al. Changes in gut microbiota control metabolic endotoxemia-induced inflammation in high-fat diet-induced obesity and diabetes in mice. Diabetes. 2008;57(6):1470-81.

36. Cani PD, Possemiers S, Van de Wiele T, Guiot Y, Everard A, Rottier O, et al. Changes in gut microbiota control inflammation in obese mice through a mechanism involving GLP-2-driven improvement of gut permeability. Gut. 2009;58(8):1091-103.

37. Saad MJ, Santos A, Prada PO. Linking Gut Microbiota and Inflammation to Obesity and Insulin Resistance. Physiology (Bethesda). 2016;31(4):283-93.

38. Shi H, Kokoeva MV, Inouye K, Tzameli I, Yin H, Flier JS. TLR4 links innate immunity and fatty acid-induced insulin resistance. J Clin Invest. 2006;116(11):3015-25

39. Nguyen MT, Favelyukis S, Nguyen AK, Reichart D, Scott PA, Jenn A, et al. A subpopulation of macrophages infiltrates hypertrophic adipose tissue and is activated by free fatty acids via Toll-like receptors 2 and 4 and JNK-dependent pathways. J Biol Chem. 2007;282(48):35279-92.

40. Saberi M, Woods NB, de Luca C, Schenk S, Lu JC, Bandyopadhyay G, et al. Hematopoietic cell-specific deletion of toll-like receptor 4 ameliorates hepatic and adipose tissue insulin resistance in high-fat-fed mice. Cell Metab. 2009;10(5):419-29.

41. Catrysse L, van Loo G. Inflammation and the Metabolic Syndrome: The Tissue-Specific Functions of NF-kappaB. Trends Cell Biol. 2017;27(6):417-29.

42. Arkan MC, Hevener AL, Greten FR, et al. IKK-beta links inflammation to obesity-induced insulin resistance. Nat Med. 2005;11(2):191-198.

43. Wang Z, Xue L, Guo C, Han B, Pan C, Zhao S, et al. Stevioside ameliorates high-fat diet-induced insulin resistance and adipose tissue inflammation by downregulating the NF-kappaB pathway. Biochem Biophys Res Commun. 2012;417(4):1280-5.

44. Weisberg SP, McCann D, Desai M, Rosenbaum M, Leibel RL, Ferrante AW, Jr. Obesity is associated with macrophage accumulation in adipose tissue. J Clin Invest. 2003;112(12):1796-808.

45. Keiran N, Ceperuelo-Mallafre V, Calvo E, Hernandez-Alvarez MI, Ejarque M, Nunez-Roa C, et al. SUCNR1 controls an anti-inflammatory program in macrophages to regulate the metabolic response to obesity. Nat Immunol. 2019;20(5):581-92.

46. Fujisaka S, Usui I, Bukhari A, Ikutani M, Oya T, Kanatani Y, et al. Regulatory mechanisms for adipose tissue M1 and M2 macrophages in diet-induced obese mice. Diabetes. 2009;58(11):2574-82.

47. Xu X, Grijalva A, Skowronski A, van Eijk M, Serlie MJ, Ferrante AW, Jr. Obesity activates a program of lysosomal-dependent lipid metabolism in adipose tissue macrophages independently of classic activation. Cell Metab. 2013;18(6):816-30.

48. Lumeng CN, Deyoung SM, Bodzin JL, Saltiel AR. Increased inflammatory properties of adipose tissue macrophages recruited during diet-induced obesity. Diabetes. 2007;56(1):16-23.

49. Haase J, Weyer U, Immig K, Kloting N, Bluher M, Eilers J, et al. Local proliferation of macrophages in adipose tissue during obesity-induced inflammation. Diabetologia. 2014;57(3):562-71.

50. Turnbaugh PJ, Hamady M, Yatsunenko T, Cantarel BL, Duncan A, Ley $\mathrm{RE}$, et al. A core gut microbiome in obese and lean twins. Nature. 2009;457(7228):480-4

51. Turnbaugh PJ, Ley RE, Mahowald MA, Magrini V, Mardis ER, Gordon JI. An obesity-associated gut microbiome with increased capacity for energy harvest. Nature. 2006:444(7122):1027-31.

52. Zhao L, Zhang F. Gut bacteria selectively promoted by dietary fibers alleviate type 2 diabetes. Science. 2018;359(6380):1151-6.

53. Song X, Zhong L, Lyu N, Liu F, Li B, Hao Y, et al. Inulin Can Alleviate Metabolism Disorders in ob/ob Mice by Partially Restoring Leptin-related Pathways Mediated by Gut Microbiota. Genomics Proteomics Bioinformatics. 2019;17(1):64-75.

54. Sivaprakasam S, Prasad PD, Singh N. Benefits of short-chain fatty acids and their receptors in inflammation and carcinogenesis. Pharmacol Ther. 2016;164:144-51. 
55. Thorburn AN, Macia L, Mackay CR. Diet, metabolites, and "western-lifestyle" inflammatory diseases. Immunity. 2014;40(6):833-42.

56. Thangaraju M, Cresci GA, Liu K, Ananth S, Gnanaprakasam JP, Browning DD, et al. GPR109A is a G-protein-coupled receptor for the bacterial fermentation product butyrate and functions as a tumor suppressor in colon. Cancer Res. 2009;69(7):2826-32.

57. Kimura I, Ozawa K, Inoue D, Imamura T, Kimura K, Maeda T, et al. The gut microbiota suppresses insulin-mediated fat accumulation via the short-chain fatty acid receptor GPR43. Nat Commun. 2013;4:1829.

58. Barczynska R, Kapusniak J, Litwin M, Slizewska K, Szalecki M. Dextrins from Maize Starch as Substances Activating the Growth of Bacteroidetes and Actinobacteria Simultaneously Inhibiting the Growth of Firmicutes, Responsible for the Occurrence of Obesity. Plant Foods Hum Nutr. 2016;71(2):190-6.

59. Valcheva R, Hotte N, Gillevet P, Sikaroodi M, Thiessen A, Madsen KL. Soluble Dextrin Fibers Alter the Intestinal Microbiota and Reduce Proinflammatory Cytokine Secretion in Male IL-10-Deficient Mice. J Nutr. 2015;145(9):2060-6. 